\title{
EMR alert cuts sepsis deaths
}

A

New York hospital has nearly halved the number of patients who die as a result of sepsis, through the creative use of its electronic medical record system.

Two years ago, Mount Sinai Hospital in New York City was struggling to catch cases of the infection-related illness early enough to save lives, says Dr. Charles Powell, chief of pulmonary and critical care medicine. The hospital's overall mortality rate and sepsis mortality rate were both unusually high compared with those at other academic medical centres in the United States. Sepsis accounted for about $50 \%$ of all deaths at Mount Sinai.

Now, the hospital's electronic medical record (EMR) system triggers a red alert whenever staff enter vital signs in a patient's chart that match the criteria for early sepsis. The alert prompts a bedside call from a team of specially trained nurse practitioners who evaluate the patient, order tests and, if necessary, jump-start treatment.

Mount Sinai has implemented this early warning and response program on eight floors beginning in 2012, with marked success. There were 77 fewer deaths from sepsis that year, representing a $40 \%$ reduction in the hospital's sepsis mortality rate compared to 2011 .

"When we began the program, the mean sepsis mortality rate was about $33 \%$... now it's at $16 \%$," which is close to the lowest rate among peer hospitals, says Powell. "We're now able to identify patients with sepsis earlier and standardize our response, and using our EMR data, we're also able to measure that response in terms of timeliness and outcomes, including transfers to intensive care and mortality."

Given that "most of the resources used were already dedicated and present at Mount Sinai," the financial cost was minimal, he says. "The only difference is how those resources get prioritized; for example, the prioritization of time to create the clinical decision support models."

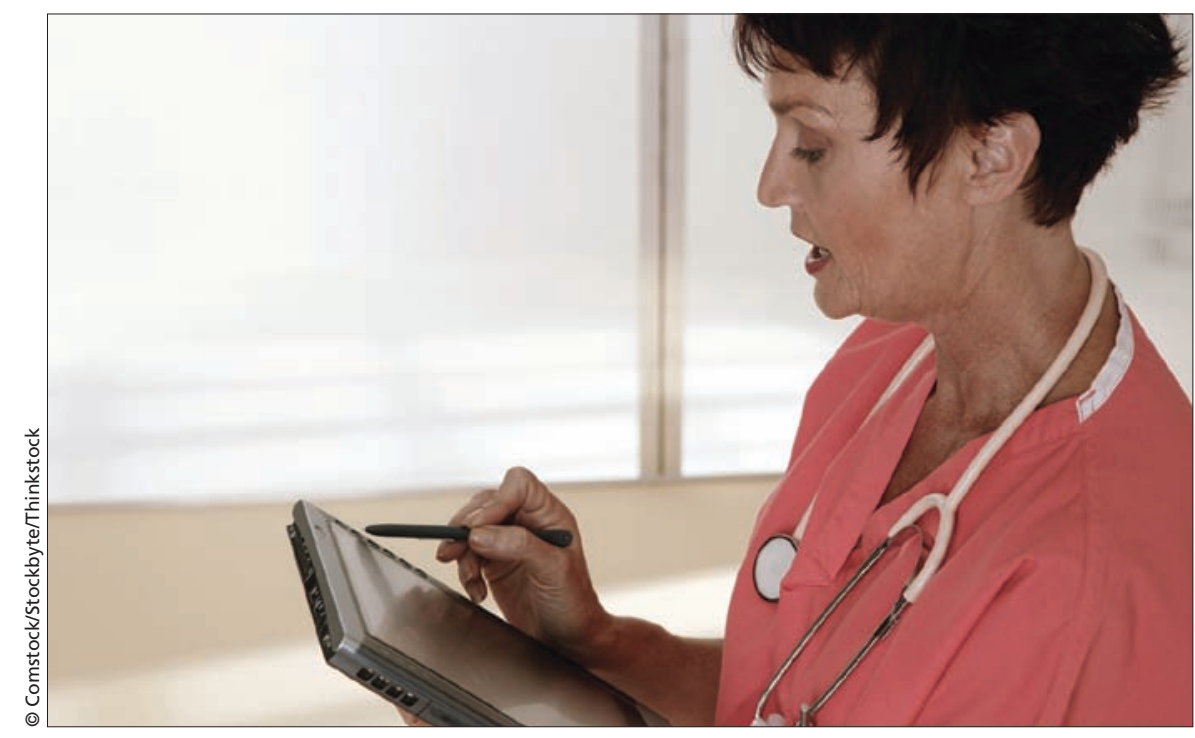

Mount Sinai Hospital's EMR system triggers a red alert whenever a patient's vital signs match the criteria for possible early sepsis.

This is good news in what has been described as a losing battle against sepsis worldwide. Hospitalizations for sepsis have more than doubled in the last decade and an estimated one-third to one-half of those patients die as a result of the condition. Wealthy countries are not exempt: the incidence of postsurgical sepsis tripled in the US between 1997 and 2006. In Canada, more than $10 \%$ of all hospital deaths are due to sepsis.

Early treatment with antibiotics and intravenous fluids can reduce the risk of death from sepsis by half. However, diagnosis is often delayed because clinical symptoms - including raised temperature and increased pulse or breathing rate - are not specific enough to provoke alarm.

Powell attributes the success of Mount Sinai's sepsis-reduction program to the fact that the EMR system doesn't dismiss those early warning signs. "There was a much greater appreciation of the seriousness that is suggested by these abnormalities in vital signs, and a significant reduction in any apprehension that may have existed before to call on an advancedlevel provider."
Judy Miranda, nursing director for transplant and rehabilitation at the hospital, says previously many nurses felt uncomfortable flagging sepsis cases on the basis of vital signs alone, and those who did often met with "push-back" from busy doctors. "Of course there are other things than sepsis that can trigger the alert, but you're only going to know if you escalate the care."

EMR alerts have lent new credence to old hunches, she says. "It meant starting fresh, changing the culture and empowering the nursing staff to know they're not giving someone a hard time by telling them they need to come and manage the patient."

According to Powell, "it's a huge lesson and cultural shift in the hospital" that's had ripples beyond the management of patients with sepsis. He says the partnership has "elevated" interactions between physicians, advancedlevel practitioners and nurses across the board. "There is enhanced appreciation for the contributions that we all bring."

Mount Sinai launched the program hospital-wide in January 2014. — Lauren Vogel, $C M A J$

CMAJ 2014. DOI:10.1503/cmaj.109-4686 